\title{
Evading the Few TeV Perturbative Limit in 3-3-1 Models
}

\author{
Alex Gomes Dias* \\ Instituto de Física, Universidade de São Paulo, \\ C. P. 66.318, 05315-970 São Paulo, SP, Brazil.
}

(Dated: October 27, 2018)

\begin{abstract}
Some versions of the electroweak $\mathrm{SU}(3)_{L} \otimes \mathrm{U}(1)_{X}$ models cannot be treated within perturbation theory at energies of few $\mathrm{TeV}$. An extended version for these models is proposed which is perturbative even at $\mathrm{TeV}$ scale posing no threatening inconsistency for test at future colliders. The extension presented here needs the addition of three octets of vector leptons, which leave three new leptonic isotriplets in the $\mathrm{SU}(2)_{L} \otimes \mathrm{U}(1)_{Y}$ subgroup. With this representation content the running of the electroweak mixing angle, $\theta_{W}(\mu)$, is such that $\sin ^{2} \theta_{W}(\mu)$ decreases with the increase of the energy scale $\mu$, when only the light states of the Standard Model group are considered. The neutral exotic gauge boson $Z^{\prime}$ marks then a new symmetry frontier.

PACS numbers: $11.10 . \mathrm{Hi} ; 12.60 . \mathrm{Cn} ;$ 12.60.Fr; $14.60 . \mathrm{Hi}$
\end{abstract}

* e-mail: alexdias@fma.if.usp.br 


\section{INTRODUCTION}

The known 3-3-1 models proposed nearly twelve years ago are good candidates to describe some new phenomena we expect to see in the near future [1]. They offer a novel phenomenology like, for example, being an appropriate framework to study in details bilepton through $e^{-} e^{-}$production in linear colliders [2], also within other observational capabilities [3, 4]. They provide a natural theoretical explanation to the families replication problem.

Despite these and other curious features the early model versions present a severe limitation. The problem is that, like in any Standard Model gauge group extension, they predict a bunch of new particles. If from one side we have a base for computing new states production, on the other side we must worry about the consequences of the additional degrees of freedom. New particles already arising at the TeV scale affect significantly the coupling evolution with energy. In fact, even if these states do not appear directly as on shell states, their degrees of freedom effectively contribute to the dynamics according to the renormalization group equations. This problem is particularly dramatic in the model versions we shall discuss here. Such versions belong to the class of 3-3-1 models where the third component in the lepton triplet is a positive charged particle [1, 5]. There the $\mathrm{SU}(3)_{L} \otimes \mathrm{U}(1)_{X}$ gauge coupling constants, denoted by $\alpha_{L}$ and $\alpha_{X}$ respectively, are linked by the following relation involving the electroweak mixing angle $\theta_{W}$

$$
\frac{\alpha_{X}(\mu)}{\alpha_{L}(\mu)}=\frac{\sin ^{2} \theta_{W}(\mu)}{1-4 \sin ^{2} \theta_{W}(\mu)} .
$$

This relation is valid above the energy scale $\mu \geq \mu_{331}$ where all symmetries become evident inside this theoretical framework. Evidently, the symmetry breakdown $\mathrm{SU}(3)_{L} \otimes \mathrm{U}(1)_{X} \rightarrow$ $\mathrm{SU}(2)_{L} \otimes \mathrm{U}(1)_{Y}$ has to be postulated to have occurred when a scalar field $\chi$ condensates with $\langle\chi\rangle \simeq \mu_{331}$. But Eq. (11) requires $\sin ^{2} \theta_{W}<1 / 4$, which is in accordance with direct measurement. Eq. (11) when confronted with the current value $\sin ^{2} \theta_{W}\left(M_{Z}\right)=0.23113(15)$ [6] gives rise to a trouble in these models. The point is that their $\mathrm{SU}(2)_{L} \otimes \mathrm{U}(1)_{Y}$ effective theory has almost the same Standard Model minimal particle content, apart from few additional scalar multiplets. Therefore, the renormalization group equation solutions in this case show us that $\sin ^{2} \theta_{W}$ increases with energy. It means that Eq. (II) points to a nonperturbative regime at energy values which we shall be just interested. The equation defines the initial value $\alpha_{X}\left(\mu_{331}\right)$ and since it comes from an abelian group it also increases with energy, being 
close to one for energies around few $\mathrm{TeV}$ in the minimal models. Consequently it is a serious problem for the perturbative approach and it could make the models less appealing.

For example, running $\sin ^{2} \theta_{W}$ with only the minimal Standard Model representation content we see that it reaches the value 0.25 at the energy scale $\mu \sim 4 \mathrm{TeV}$ [7]. A study of perturbative limits in 3-3-1 models was carried out in [8]. There it was shown that perturbation theory cannot be used already above scales of few $\mathrm{TeV}$ if we consider the minimal versions with or without supersymmetry.

There are some 3-3-1 constructions which do not have the problem above [9]. But the ones considered here account for doubly charged bileptons intermediating processes where partial lepton number is violated, representing a very distinct signal to search for [2, 3]. Other aspects and recent work on these models can be found in Ref. [10].

In this work it is proposed an additional matter content to the early 3-3-1 models where perturbative treatment can be applied without such tight restrictions. We see that the simplest representations to be added to the minimal versions are four octets, three of vectorial leptons plus and a scalar one. With them there is a prediction of a decreasing behavior for $\sin ^{2} \theta_{W}$ in a certain range of energy. The new exotic leptons are interesting also from the phenomenological point of view under the Standard Model gauge group in the see-saw mechanism [11], and also under the extended group we consider here [12].

The work is organized as follows. In Sec. Iwe study the renormalization group equations to understand how the nonperturbative limit appears in the minimal models. We then define a condition which should be satisfied in the model extensions, in order to make them perturbatively safe. We, then, justify the choice of the new representations content to be introduced. In Sec. III we show how the perturbative limit is raised in two 3-3-1 models. In Sec. IV it is suggested a possible decoupling off all the scalars for energies below $\mu_{331}$. We finish in Sec. $\nabla$ with the discussion of our results.

\section{THE RUNNING OF THE ELECTROWEAK MIXING ANGLE}

At the $\mathrm{SU}(2)_{L} \otimes \mathrm{U}(1)_{Y}$ energy scale the renormalization group equations dictating the running of the gauge couplings at one loop level are given by

$$
\frac{1}{\alpha_{i}(\mu)}=\frac{1}{\alpha_{i}\left(M_{Z}\right)}+\frac{1}{2 \pi} b_{i} \ln \left(\frac{M_{Z}}{\mu}\right), \mu \leq \mu_{331}
$$


with $i=1,2$ and $\alpha_{2}, \alpha_{1}$ are the coupling constants of the $\mathrm{SU}(2)_{L}, \mathrm{U}(1)_{Y}$ groups, respectively. The third equation involving the QCD coupling constant is irrelevant for our purposes here at this approximation level, and so it will not enter in our developments. As we have said above, for posterior use in the context of the 3-3-1 models we denote the gauge coupling constants $\alpha_{L}, \alpha_{X}$ for the groups $\mathrm{SU}(3)_{L}, \mathrm{U}(1)_{X}$, respectively. In a generic gauge group the $b_{i}$ coefficients are given by

$$
b_{i}=\frac{2}{3} \sum_{\text {fermions }} T_{R}(F)_{i}+\frac{1}{3} \sum_{\text {scalars }} T_{R}(S)_{i}-\frac{11}{3} C_{2}(G)_{i}
$$

for Weyl fermions and complex scalars, with the generators $T^{a}$ satisfying $\operatorname{Tr}\left[T^{a}(I) T^{b}(I)\right]=$ $T_{R}(I) \delta^{a b}$ where $I=F, S$. For $S U(N) T_{R}(I)=1 / 2$ in the fundamental representation and $C_{2}(G)=N . C_{2}(G)=0$ for $U(1)$. These numbers can be computed for other different representations with aid of the identity $T_{R}(I)\left(N^{2}-1\right)=C_{2 R}(I) d_{R}(I)$, where $C_{2 R}(I)$ is the quadratic Casimir operator and $d_{R}(I)$ is the representation dimension. For $U(1)_{y}$ we use $\sum T_{R 1}(F, S)=\sum y^{2}$ where $y=Y / 2$ for the Standard Model and $y=X$ for the 3-3-1 models.

Below $\mu_{331}$ the $\sin ^{2} \theta_{W}$ running is then, according to the Standard Model gauge group,

$$
\sin ^{2} \theta_{W}(\mu)=\frac{1}{1+\frac{\alpha_{2}(\mu)}{\alpha_{1}(\mu)}}, \mu \leq \mu_{331}
$$

We see that for a $\mathrm{SU}(2)$ content presenting asymptotic freedom, i. e. $\alpha_{2} \rightarrow 0$ as $\mu \rightarrow \infty$, $\sin ^{2} \theta_{W}$ increases with the energy. Above the scale $\mu_{331}$ we run the renormalization group equations with particles in the full $\mathrm{SU}(3)_{L} \otimes \mathrm{U}(1)_{N}$ representations. In the minimal models when we consider $\mu_{331} \leq 2 \mathrm{TeV}$, then perturbative expansion in $\alpha_{X}$ makes sense only at energy values lower than $2.4 \mathrm{TeV}$ [8]. It jeopardizes the perturbative analysis of the neutral exotic vector boson $Z^{\prime}$ which is one of the model predictions, since its mass is naively expected to have a lower limit near this perturbative upper bound.

We observe that for a $\mathrm{SU}(2)$ content not presenting asymptotic freedom, i. e. with $\alpha_{2}(\mu)$ growing when $\mu \rightarrow \mu_{331}$, then $\sin ^{2} \theta_{W}(\mu)$ decreases in the same energy interval improving the perturbative validity in such models. From Eqs. (2) and (4) it is deduced that the condition for accomplishing this is that

$$
b_{2}>\tan ^{2} \theta_{W}\left(M_{Z}\right) b_{1}
$$

Inserting the $\tan ^{2} \theta_{W}$ value at the $Z$ pole, the condition between the coefficients turns out to be $b_{2}>0.3 b_{1}$. So, if we want to satisfy it some additional fields in nontrivial $\mathrm{SU}(2)$ 
representations must be considered. In order to give a bigger contribution to $b_{2}$ than to $b_{1}$ their hypercharge must be small.

To be more explicit we take the 3-3-1 model with only three scalar triplets as our first representative of the class [5]. The electric charge operator $\mathcal{Q}$ is,

$$
\mathcal{Q}=\frac{1}{2}\left(\lambda_{3}-\sqrt{3} \lambda_{8}\right)+X
$$

The representation content in the quark sector is (the numbers inside parenthesis mean their transformation properties under $\mathrm{SU}(3)_{C}, \mathrm{SU}(3)_{L}$ and $\mathrm{U}(1)_{X}$ respectively, or under $\mathrm{SU}(3)_{C}$, $\mathrm{SU}(2)_{L}$ and $\mathrm{U}(1)_{Y}$ when this is the case) $Q_{m L}=\left(d_{m}, u_{m}, j_{m}\right)_{L}^{T} \sim\left(\mathbf{3}, \mathbf{3}^{*},-1 / 3\right), m=1,2$; $Q_{3 L}=\left(u_{3}, d_{3}, J\right)_{L}^{T} \sim(\mathbf{3}, \mathbf{3}, 2 / 3)$; and the respective right-handed components in singlets $u_{\beta R} \sim(\mathbf{3}, \mathbf{1}, 2 / 3), d_{\beta R} \sim(\mathbf{3}, \mathbf{1},-1 / 3), \beta=1,2,3 ; J_{R} \sim(\mathbf{3}, \mathbf{1}, 5 / 3)$ and $j_{m R} \sim(\mathbf{3}, \mathbf{1},-4 / 3)$.

In the scalar sector the three triplets are $\chi=\left(\chi^{-}, \chi^{--}, \chi^{0}\right)^{T} \sim(\mathbf{1}, \mathbf{3},-1), \rho=$ $\left(\rho^{+}, \rho^{0}, \rho^{++}\right)^{T} \sim(\mathbf{1}, \mathbf{3}, 1)$ and $\eta=\left(\eta^{0}, \eta_{1}^{-}, \eta_{2}^{+}\right)^{T} \sim(\mathbf{1}, \mathbf{3}, 0)$.

Finally, in this model, leptons come in triplets like

$$
\Psi_{a L}=\left(\nu_{a}, l_{a}, E_{a}\right)_{L}^{T} \sim(\mathbf{1}, \mathbf{3}, 0)
$$

where $a=e, \mu, \tau$. With their right-handed components in singlets $l_{a R} \sim(\mathbf{1}, \mathbf{1},-1), E_{a R} \sim$ $(\mathbf{1}, \mathbf{1},+1)$. We have omitted right-handed neutrinos since, like any neutral singlet representation, they are not relevant for our purposes here. The effective $\mathrm{SU}(2)_{L} \otimes \mathrm{U}(1)_{Y}$ theory coming from this model, when the condensation $\left\langle\chi^{0}\right\rangle=w / \sqrt{2}$ occurs, have the same light multiplets which dominate the spectrum of the Standard Model plus an extra scalar isodoublet $\eta=\left(\eta^{0}, \eta_{1}^{-}\right)^{T} \sim(\mathbf{1}, \mathbf{2},-1)$. The value $w / \sqrt{2}$ is going to be identified with $\mu_{331}$.

Next we consider the renormalization group coefficients in Eq. (3) at the $\mathrm{SU}(2)_{L} \otimes \mathrm{U}(1)_{Y}$ level with three usual matter generations, taking into account at most the two nontrivial lowest dimensional SU(2) representations, i. e., isodoublets and isotriplets. We reserve the prefix $i$ so just for when we talk about $\mathrm{SU}(2)_{L} \otimes \mathrm{U}(1)_{Y}$ representations. Let $N_{S}$ be the number of scalar isodoublets, $N_{F}$ the number of new exotic fermionic isodoublets, $N_{T S}$ the number of scalar isotriplets and $N_{T F}$ the number of new exotic fermionic isotriplets, with $Y$ being their respective hypercharge, then from Eq. (3)

$$
\begin{aligned}
b_{1} & =\frac{1}{3}\left(Y_{F}^{2} N_{F}+\frac{Y_{S}^{2}}{2} N_{S}+20\right)+\frac{1}{2}\left(Y_{T F}^{2} N_{T F}+\frac{Y_{T S}^{2}}{2} N_{T S}\right), \\
b_{2} & =\frac{1}{3}\left(N_{F}+\frac{1}{2} N_{S}+4 N_{T F}+2 N_{T S}-10\right) .
\end{aligned}
$$


When dealing with fermions in vectorial representations $N_{F}$ and $N_{T F}$ must be multiplied by 2 . In the model above we have only two $Y_{S}^{2}=1$ scalar isodoublets to be considered, i. e. $N_{S}=2$, in the Eqs. (17) and (8) so that $\left(b_{1}, b_{2}\right)=(7,-3)$. And it is not possible to satisfy the condition in (5). In fact the $\sin ^{2} \theta_{W}$ running shows that the value 0.25 is reached at $4.1 \mathrm{TeV}$ as we see in Fig. (11) (where for future reference it is also shown the same running for the model with a scalar sextet). The scale $\mu_{331}$ is then below this value and so the energy $\Lambda$ at which the pole is attained is such that $\Lambda \leq 4.1 \mathrm{TeV}$. The Landau-like pole behavior with $\mu_{331}$ has been studied in Ref. [8]. For example, if $\mu_{331}=1 \mathrm{TeV}$ we have that at energies $E \simeq 1.9 \mathrm{TeV}, \alpha_{X}>1$ and $M_{Z^{\prime}} \gtrsim 1.9 \mathrm{TeV}$ so that the model looses its perturbative character below the exotic neutral vector boson $Z^{\prime}$ mass. In part this is due to the fact that the right handed components of the exotic quarks, $J$ and $j_{m}$, necessary to complete the $\mathrm{SU}(3)_{L}$ representations have a value of the $\mathrm{U}(1)_{X}$ charge larger than the other particles. Thus, they are the major contribution to the $\alpha_{X}$ running. Under the assumption that they are much above the $Z^{\prime}$ scale, the perturbative limit is pushed a little above. But this brings these quarks out of experimental reach. We would like to pursue the possibility of keeping them observable at $\mathrm{TeV}$ scale. To make the exotic quarks very heavy means that the $\operatorname{VEV}\left\langle\chi^{0}\right\rangle$ is very high and since this is also responsible for giving mass to all new fermions in the $\mathrm{SU}(3)_{L}$ triplets and the new gauge bosons, it would bring almost all the predictions of the model out of experimental reach. Of course, this is not interesting from the phenomenological point of view.

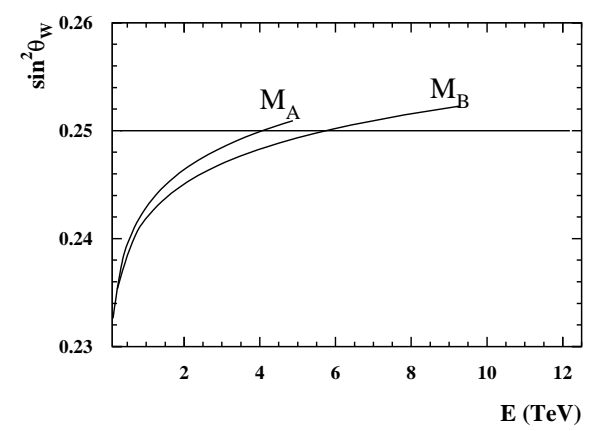

FIG. 1: Running of the electroweak mixing angle for the minimal models considering only light degrees of freedom under the Standard Model subgroup. Model A with three scalar triplets, and model B, the same as A with a scalar sextet added. 
We see from Eqs. (17) and (8) that multiplets having large hypercharge take us in the opposite direction of satisfying (5). Isodoublets of vectorial leptons or/and scalars with zero hypercharge could then be considered. But the number of them needed would be, at least, eight of vectorial leptons or thirty two of scalars with components having electric charge $1 / 2$ and $-1 / 2$.

The simplest solution is to have exotic isotriplets. Preferentially fermionics. From Eqs. (77) and (88) one can also see that the minimal number of isotriplets are three, all of vectorial leptons with $Y=0$. They are written as

$$
\overrightarrow{\mathcal{T}}=\left(\begin{array}{cc}
\frac{1}{\sqrt{2}} t^{0} & t^{+} \\
t^{-} & -\frac{1}{\sqrt{2}} t^{0}
\end{array}\right),
$$

transforming as $(\mathbf{1}, \mathbf{3}, 0)$ under the Standard Model group. They must be color singlets, otherwise QCD asymptotic freedom would be lost. This isotriplet couples with the usual matter. It has interesting consequences in the see-saw mechanism and it does not represent cosmological troubles if they belong to the electroweak scale, as it was studied along with other phenomenological aspects in [11]. To be consistent with the $Z^{0}$ decay width, their components must be heavier than $M_{Z^{0}} / 2$.

Now the lowest dimensional $\mathrm{SU}(3)$, with $\mathrm{U}(1)_{N}$ charge zero, representations which contain an isotriplet decomposes under the Standard Model $\mathrm{SU}(2) \otimes \mathrm{U}(1)_{Y}$ group as

$$
\begin{aligned}
& \mathbf{6}=\mathbf{3}_{-2} \oplus \mathbf{2}_{1} \oplus \mathbf{1}_{4}, \\
& \mathbf{8}=\mathbf{3}_{0} \oplus \mathbf{2}_{-3} \oplus \mathbf{2}_{3} \oplus \mathbf{1}_{0} .
\end{aligned}
$$

The subscripts refer to their hypercharge value $Y$. The sextet gives rise to a $Y^{2}=4$ isotriplet which could also do the job but it has a $Y^{2}=16$ isosinglet. It could be assumed to be heavy not affecting the spectra below $\mu_{331}$. But we want to avoid such an assumption. It would be needed also three sextets of fermions if the $Y^{2}=16$ singlet components were heavy, plus a scalar one to generate masses. The octet on the other hand has the advantage of having an isosinglet and an isotriplet both neutrals in hypercharge which, therefore, do not contribute to $b_{1}$. But it has also two isodoublets with $Y^{2}=9$. They can be made heavy if a scalar octet is present and it condensates at the $\mu_{331}$, as we are going to discuss below. We shall then consider here the exotic vectorial leptons in three fermionic octets since they are the simplest solution. With them we also do not need to concern with anomalies. They have 
the form

$$
\Xi_{a}=\left(\begin{array}{ccc}
\frac{1}{\sqrt{2}} t_{a}^{0}+\frac{1}{\sqrt{6}} \lambda_{a}^{0} & t_{a}^{+} & \delta_{a}^{-} \\
t_{a}^{-} & -\frac{1}{\sqrt{2}} t_{a}^{0}+\frac{1}{\sqrt{6}} \lambda_{a}^{0} & \delta_{a}^{--} \\
\xi_{a}^{+} & \xi_{a}^{++} & -\frac{2}{\sqrt{6}} \lambda_{a}^{0}
\end{array}\right)
$$

transforming as $(\mathbf{1}, \mathbf{8}, 0)$, where $a=1,2,3$.

When it is introduced a representation like this, with all spinor components of the matter fields belonging to the same multiplet, differently from the usual chiral constructions, gauge invariance does allow for initial mass terms for the fields in these representations. Therefore, there would be a bare mass term like $M_{a b} \operatorname{Tr} \overline{\Xi_{a}} \Xi_{b}$ in the Lagrangian in its symmetric fase.

To satisfy the condition in Eq. (15) the $Y^{2}=9$ lepton isodoublets $\delta=\left(\delta^{-}, \delta^{--}\right)^{T}$ and $\xi=\left(\xi^{++}, \xi^{+}\right)^{T}$ in Eq. (10) must then be decoupled from energies below $\mu_{331}$. This can be achieved with an octet representation of scalars similar to the fermions above. Calling this representation $\Sigma$ its form is

$$
\Sigma=\left(\begin{array}{ccc}
\frac{1}{\sqrt{2}} \phi^{0}+\frac{1}{\sqrt{6}} \varphi^{0} & \phi^{+} & \varphi_{1}^{-} \\
\phi^{-} & -\frac{1}{\sqrt{2}} \phi^{0}+\frac{1}{\sqrt{6}} \varphi^{0} & \varphi_{1}^{--} \\
\varphi_{2}^{+} & \varphi_{2}^{++} & -\frac{2}{\sqrt{6}} \varphi^{0}
\end{array}\right)
$$

transforming as $(\mathbf{1}, \mathbf{8}, 0)$. To see how the decoupling can be worked out let us analyze the mass spectra for these new fields.

The Yukawa interactions in the lepton sector are

$$
\begin{aligned}
-\mathcal{L}_{Y} & =H_{a b} \epsilon_{i j k} \overline{\left(\Psi_{i a L}\right)^{c}} \Psi_{j b L} \eta_{k}+\bar{\Psi}_{a L}\left(G_{a b}^{l} l_{b R} \rho+G_{a b}^{E} E_{b R} \chi\right) \\
& +G_{a b}^{\Xi} \bar{\Psi}_{a L} \Xi_{b} \eta+\frac{1}{2} \operatorname{Tr}\left[\bar{\Xi}_{a}\left(A_{a b} \Xi_{b} \Sigma+B_{a b} \Sigma \Xi_{b}+M_{a b} \Xi_{b}\right)\right] \\
& + \text { H.c. }
\end{aligned}
$$

where repeated indices mean summation. $H_{a b}$ is an antisymmetric matrix with $G_{a b}^{l}, G_{a b}^{E}, G_{a b}^{\Xi}$ general complex matrices and $A_{a b}, B_{a b}, M_{a b}$ real symmetric matrices which will be assumed diagonal for simplicity.

Then $Y^{2}=9$ isodoublets in $\Xi_{a}$ decouple when $\langle\Sigma\rangle=\frac{1}{\sqrt{6}}\left\langle\varphi^{0}\right\rangle \operatorname{diag}(1,1,-2)$, when the following relations are satisfied

$$
\left(A_{a b}+B_{a b}\right) \frac{\left\langle\varphi^{0}\right\rangle}{\sqrt{6}}+M_{a b} \simeq m_{S M}
$$




$$
\begin{aligned}
& m_{S M}-3 A_{a b} \frac{\left\langle\varphi^{0}\right\rangle}{\sqrt{6}} \simeq \mu_{331} \\
& m_{S M}-3 B_{a b} \frac{\left\langle\varphi^{0}\right\rangle}{\sqrt{6}} \simeq \mu_{331} .
\end{aligned}
$$

Where $m_{S M}$ is a mass scale belonging to the Standard Model mass spectrum. The first relation above leaves the isotriplets $\overrightarrow{\mathcal{T}}_{a}$ in $\Xi_{a}$ with appropriate masses below $\mu_{331}$. The last two relations in (13) ensure that the masses of the components of the isodoublets $\delta$ and $\xi$ are heavy enough, since $\langle\Sigma\rangle$ breaks the electroweak $\mathrm{SU}(3)_{L}$ symmetry down to the $\mathrm{SU}(2)_{L}$ group $\left\langle\varphi^{0}\right\rangle \simeq \mu_{331}$ and the isodoublets are, in this way, decoupled of the Standard Model particle mass spectrum. The isosinglets $\lambda_{a}^{0}$ in the octet $\Xi_{a}$ have then masses of order $m_{\lambda_{a}^{0}} \simeq M_{a a}-m_{S M}$ which is the same order of $\mu_{331}$ according to the Eqs. (13). The simple analysis presented here is not complete but it is sufficient for the purposes of showing

that the higher hypercharge fields can be heavy. Thus, only the vector lepton isotriplets $\overrightarrow{\mathcal{T}}_{a}$ together with the known fields contribute to the renormalization group equations at energies $E<\mu_{331}$.

In the last stage of symmetry breakdown when the Standard Model group reduces to the electromagnetic abelian gauge group, $\mathrm{U}(1)_{e m}$, we see from the term $G_{a b}^{\Xi} \bar{\Psi}_{a L} \Xi_{b} \eta$ in Eq. (12) that there will be a mixing between the know and the exotic leptons. It could be avoided by imposing some sort of discrete symmetry. Their phenomenological consequences will be carried out elsewhere.

Finally, we should say that condensation of $\phi^{0}$ in $\Sigma$ is very constrained by the $\rho$ parameter relating the $W^{ \pm}$and $Z$ masses.

We are ready now to show how the perturbative limit is improved with these representations.

\section{THE PERTURBATIVE LIMIT}

We now turn out to look how the perturbative limit is characterized by the scale $\mu_{331}$ breaking the $\mathrm{SU}(3)_{L} \otimes \mathrm{U}(1)_{X}$ symmetry. The relevant running equation is that of the coupling constant $\alpha_{X}$ coming from the abelian factor $\mathrm{U}(1)_{X}$. This running is

$$
\frac{1}{\alpha_{X}(\mu)}=\frac{1}{\alpha_{X}\left(\mu_{331}\right)}+\frac{1}{2 \pi} b_{X} \ln \left(\frac{\mu_{331}}{\mu}\right), \mu \geq \mu_{331} .
$$


$b_{X}$ is computed considering the fields in the full 3-3-1 representation. Eq. (14) starts with the value $\alpha_{X}\left(\mu_{331}\right)$ which can be determined using Eqs. (11) and (4) and the fact that $\alpha_{2}\left(\mu_{331}\right)=\alpha_{L}\left(\mu_{331}\right)$. That is,

$$
\frac{1}{\alpha_{X}\left(\mu_{331}\right)}=\frac{1}{\alpha_{1}\left(\mu_{331}\right)}-\frac{3}{\alpha_{2}\left(\mu_{331}\right)} .
$$

Perturbative expansion does not make sense any more when a scale $\mu=M^{\prime}$ such that $\alpha_{X}\left(M^{\prime}\right)=1$ is reached. We have then

$$
M^{\prime}=\mu_{331}\left[\frac{M_{Z}}{\mu_{331}}\right]^{\frac{b_{1}-3 b_{2}}{b_{X}}} e^{\frac{2 \pi}{b_{X}}\left[\frac{1}{\alpha\left(M_{Z}\right)}\left(1-4 \sin ^{2} \theta_{W}\left(M_{Z}\right)\right)-1\right],}
$$

$\alpha\left(M_{Z}\right)=1 / 128$ is the fine structure constant at the $Z$ pole, with $M_{Z}=91.2 \mathrm{GeV}$. We can now see how the perturbative limit is changed with $\mu_{331}$.

The additional representations to be considered are then the three leptonic $\mathrm{SU}(3)_{L}$ octets $\Xi_{a},(a=1,2,3)$, like (10) which leave three leptonic isotriplets like (9) at energies $E \leq \mu_{331}$, i. e. $N_{T F}=3$ in Eqs. (17) and (8) . The scalars in $\Sigma$ can be assumed to contribute with a $Y^{2}=0$ isotriplet and two $Y^{2}=9$ isodoublets. Thus from Eqs. (17) and (8) we have $\left(b_{1}, b_{2}\right)=(10,6)$, which obviously satisfies the condition (5). In Fig. (2) the $\sin ^{2} \theta_{W}$ running in this model is shown(again for future reference it is also shown the curves in the model with a scalar sextet).

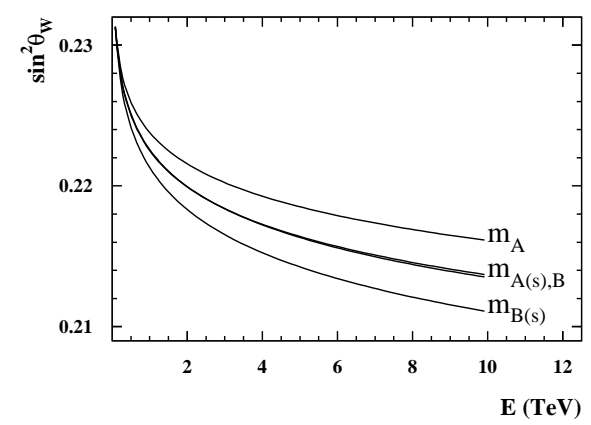

FIG. 2: Running of the electroweak mixing angle for the extended models for energies $\mathrm{E} \leq \mu_{331}$. Model A, $m_{A}$, with only three scalar triplets, and model $\mathrm{B}, m_{B}$, with a scalar sextet. (s) means that the $Y^{2}=9$ scalars are disregarded. The models $m_{A}(s)$ and $m_{B}$ have degenerate curves.

From Eq. (3) we also have $b_{X}=26$. For example, if $\mu_{331}=1 \mathrm{TeV}$ then $M^{\prime}=16.8$ $\mathrm{TeV}$; if $\mu_{331}=3 \mathrm{TeV}$ then $M^{\prime}=70.6 \mathrm{TeV}$. Disregarding the $Y^{2}=9$ scalar isodoublets 
$\left(b_{1}, b_{2}\right)=(7,17 / 3)$ and if $\mu_{331}=1 \mathrm{TeV}$ then $M^{\prime}=20.2 \mathrm{TeV}$; if $\mu_{331}=3 \mathrm{TeV}$ then $M^{\prime}=92.3$ $\mathrm{TeV}$. The perturbative limit is still improved in this case because $b_{1}$ is lowered. We see then that the model is made perturbatively much more viable with the addition of octet representations.

\section{A. The model with a scalar sextet}

Another representation content in the leptonic sector is possible in accordance with the electric charge operator Eq. (6) . The positron and his cousins occupying the third component of the leptonic triplets which are represented by [1],

$$
\Psi_{a L}=\left(\nu_{a}, l_{a}, l_{a}^{c}\right)_{L}^{T} \sim(\mathbf{1}, \mathbf{3}, 0)
$$

with $a=e, \mu, \tau$. A scalar sextet

$$
S=\left(\begin{array}{ccc}
\sigma_{1}^{0} & h_{1}^{-} & h_{2}^{+} \\
h_{1}^{-} & H_{1}^{--} & \sigma_{2}^{0} \\
h_{2}^{+} & \sigma_{2}^{0} & H_{2}^{++}
\end{array}\right)
$$

transforming as $(\mathbf{1}, \mathbf{6}, 0)$ is necessary in the minimal representation content to generate all the lepton masses when $\left\langle\sigma_{2}^{0}\right\rangle \neq 0 .\left\langle\sigma_{1}^{0}\right\rangle \neq 0$ could give a Majorana mass to the neutrinos.

The Yukawa interactions in the lepton sector are now

$$
\begin{aligned}
-\mathcal{L}_{Y}^{s} & =H_{a b} \epsilon_{i j k} \overline{\left(\Psi_{i a L}\right)^{c}} \Psi_{j b L} \eta_{k}+G_{a b}^{\Psi} \bar{\Psi}_{a L} S\left(\Psi_{i b L}\right)^{c} \\
& +G_{a b}^{\Xi} \bar{\Psi}_{a L} \Xi_{b} \eta+F_{a b} \epsilon_{i j k} \overline{\Psi_{i a L}}\left(S^{\dagger} \Xi_{b}\right)_{j k} \\
& +\frac{1}{2} \operatorname{Tr}\left[\overline{\Xi_{a}}\left(A_{a b} \Xi_{b} \Sigma+B_{a b} \Sigma \Xi_{b}+M_{a b} \Xi_{b}\right)\right] \\
& + \text { H.c. }
\end{aligned}
$$

And the same decoupling of the $Y^{2}=9$ lepton doublets can be worked out as before. Mixing between the know and exotic leptons happens through $G_{a b}^{\Xi} \bar{\Psi}_{a L} \Xi_{b} \eta$ and $F_{a b} \epsilon_{i j k} \overline{\Psi_{i a L}}\left(S^{\dagger} \Xi_{b}\right)_{j k}$ in this case. As before, it could be avoided by imposing some sort of discrete symmetry.

This model has the following additional multiplets under the $\mathrm{SU}(3)_{C} \otimes \mathrm{SU}(2)_{L} \otimes \mathrm{U}(1)_{Y}$ symmetry besides those in the three triplets model: one isodoublet $\left(h_{2}^{+}, \sigma_{2}^{0}\right) \sim(\mathbf{1}, \mathbf{2},+1)$, a nonhermitian isotriplet $\left(H_{1}^{--}, h_{1}^{-}, \sigma_{1}^{0}\right) \sim(\mathbf{1}, \mathbf{3},-2)$, and an isosinglet $H_{2}^{++} \sim(\mathbf{1}, \mathbf{1},+2)$. Then, at energies below $\mu_{331}$ three scalar isodoublets with $Y^{2}=1$ and two scalar isodoublets 
with $Y^{2}=9$ are taken into account but now we have one more scalar isotriplet plus that coming from the scalar octet. The other scalar fields including the isosinglet $H_{2}^{++}$are taken into account only at energies above $\mu_{331}$. In this case the inclusion of the isosinglet $H_{2}^{++}$does not affect so significantly the running below $\mu_{331}$. Therefore, $N_{S}=3$ with $Y^{2}=1, N_{S}=2$ with $Y^{2}=9, N_{T S}=1$ with $Y^{2}=4, N_{T S}=1$ with $Y^{2}=0$ and $N_{T F}=3$ with $Y^{2}=0$. So that from Eqs. (77) and (8) we have $\left(b_{1}, b_{2}\right)=(67 / 6,41 / 6)$. Obviously, this satisfies the condition (5). From Eq. (32) we also have $b_{X}=22$ since there is no right-handed charged leptons to be counted. For example, if $\mu_{331}=1 \mathrm{TeV}$ then $M^{\prime}=32.4 \mathrm{TeV}$; if $\mu_{331}=3 \mathrm{TeV}$ then $M^{\prime}=155.0 \mathrm{TeV}$. Disregarding the $Y^{2}=9$ scalar isodoublets coming from the octet $\Sigma,\left(b_{1}, b_{2}\right)=(49 / 6,13 / 2)$, so if $\mu_{331}=1 \mathrm{TeV}$ then $M^{\prime}=40.3 \mathrm{TeV}$; if $\mu_{331}=3 \mathrm{TeV}$ then $M^{\prime}=213.0 \mathrm{TeV}$. Again, perturbative limit is still improved in this case since $b_{1}$ is lowered.

\section{POSSIBLE DECOUPLING OF ALL SCALARS AT ENERGIES $E<\mu_{331}$}

In the preceding discussions it was considered that some scalar multiplets remain light enough, i. e., with masses much below the $\mu_{331}$ scale, so that they could participate in the counting for the coefficients in Eqs. (77) and (8). It only happens if there is a sort of fine tuning among the scalar self-interaction couplings. The reason for this comes from the fact that the scalar octet has interactions with all the scalar multiplets. The scalar potential for the three triplets model plus the octet is then

$$
\begin{aligned}
V & =V_{T}+m_{3} \eta^{\dagger} \Sigma \eta+m_{4} \rho^{\dagger} \Sigma \rho+m_{5} \chi^{\dagger} \Sigma \chi \\
& +\operatorname{Tr}\left[\Sigma^{2}\left(\lambda_{1} \Sigma^{2}+m_{1} \Sigma-m_{2}^{2}\right)\right]+\lambda_{2}\left(\operatorname{Tr}\left[\Sigma^{2}\right]\right)^{2} \\
& +\operatorname{Tr}\left[\Sigma^{2}\right]\left(\lambda_{3} \eta^{\dagger} \eta+\lambda_{4} \rho^{\dagger} \rho+\lambda_{5} \chi^{\dagger} \chi\right) .
\end{aligned}
$$

Where $V_{T}$ stands for the collection of all terms involving only $\eta, \rho$ and $\chi$. Note that we cannot impose invariance under $\Sigma \rightarrow-\Sigma$, since it would also forbid the interaction between $\Sigma$ and the $\Xi_{a}$ 's preventing the mechanism of decoupling the lepton isodoublets $\delta$ and $\xi$ of the Standard Model scale. The last term in this potential gives a quadratic mass term of order $\left\langle\varphi^{0}\right\rangle^{2}$ for the triplets unless $\lambda_{3}, \lambda_{4}$ and $\lambda_{5}$ are fine tuned. Thus, it can happen that no single scalar be part of the spectrum below $\mu_{331}$. In fact, the only massive scalar state which could be free of mass contributions coming from $\langle\Sigma\rangle$ would belong to the neutral scalar imaginary part, the pseudoscalar. But in the minimal model, i. e., without regarding the octets, there 
is just one massive pseudoscalar and it might be heavy, since its mass is of order $\left\langle\chi^{0}\right\rangle$. Of course, careful mass spectral analysis should be done but the arguments we just have given indicate that it really should happen. Because of the reason that the major contributions to the renormalization group equations are due to the fermions, it is not expected that the perturbative limit will change significantly when the scalars are omitted below $\mu_{331}$. But to produce any fundamental scalar would require energies $E \geq \mu_{331}$.

\section{DISCUSSION}

We have shown that the addition of octet representations can turn 3-3-1 models perturbatively viable at the upcoming TeV energy scale. The main effect is that they predict a running to the electroweak angle, or better $\sin ^{2} \theta_{W}$, such that it decreases with the increasing of the energy until the scale $\mu_{331}$ which characterizes the appearance of a $\mathrm{SU}(3)_{L} \otimes \mathrm{U}(1)_{X}$ electroweak symmetry. It could be tested providing a direct test of exclusion for the extended models we have presented here. With the electroweak angle evolving in this way the $\mathrm{U}(1)_{X}$ coupling constant, $\alpha_{X}$, starts to run with a value which is lower than the one appearing in the minimal models. From this point $\sin ^{2} \theta_{W}$ begins to increase with energy but it will reach values near 0.25 at energies bigger than tens of $\mathrm{TeV}$.

The same behavior could be accomplished with other representations different from octets. But these representations would be in general bigger than the ones we have considered here, and they would need more complicated mechanisms to decouple isomultiplets with large hypercharge. If instead we consider exotic leptons in sextets it is necessary to assume that the isosinglets with $Y^{2}=4$ belonging to them are decoupled at energies $E \leq \mu_{331}$. Thus octets are simpler and more convenient.

Although the additional representations turn the $\mathrm{SU}(3)_{L}$ gauge coupling, $\alpha_{3 L}$, looses its asymptotic freedom, it becomes bigger than one at energies much higher than $M^{\prime}$. Thus, it does not represent a problem.

Symmetry breakdown at the scale $\mu_{331}$ is marked with the appearance of four new charged vector bosons, two of them carrying two units of electric charge, and the exotic neutral one $Z^{\prime}$. Different from the exotic charged vector bosons, this neutral one does not receive 
contribution to its mass due to the scalar octet and it is bounded according to

$$
M_{Z^{\prime}} \gtrsim_{2}\left[\alpha_{2}\left(\mu_{331}\right) \frac{1-\sin ^{2} \theta_{W}\left(\mu_{331}\right)}{1-4 \sin ^{2} \theta_{W}\left(\mu_{331}\right)}\right]^{\frac{1}{2}} \mu_{331} .
$$

This is valid for both models here. It is assumed that $\sqrt{2}\left\langle\chi_{0}\right\rangle \simeq \mu_{331}$, which is quite reasonable since as $\langle\Sigma\rangle,\langle\chi\rangle$ also breaks the symmetry down to the Standard Model group. $M_{Z^{\prime}}$ grows then linearly with $\mu_{331}$ so that its production will mark, according to these models, the scale at which $\mathrm{SU}(3)_{W}$ should be taken into account. Thus, the $Z^{\prime}$ boson would have a mass which could be tens of $\mathrm{TeV}$ or even much less, if other phenomenological constraints allow for it.

Finally, we should say that supersymmetry does not relieve the perturbative limit in the minimal model versions [8]. It brings more degrees of freedom with the supersymmetric partners and so it worsens the problem. But for the extended models presented here it is expected that it makes the value of $M^{\prime}$ in Eq. (16) increase.

The author thanks C. A de S. Pires, P. S. R. da Silva and V. Pleitez for their useful comments and M. Caballero for reading the manuscript. This work has been supported by FAPESP under the process 01/13607-3.

[1] F. Pisano and V. Pleitez, Phys. Rev. D 46 (1992) 410; R. Foot, O. F. Hernandez, F. Pisano and V. Pleitez, Phys. Rev. D 47 (1993) 4158; P. H. Frampton, Phys. Rev. Lett. 69 (1992) 2889.

[2] P. H. Frampton and A. Rasin, Phys. Lett. B482, 129 (2000).

[3] B. Dion, T. Gregoire, D. London, L. Marleau and H. Nadeau, Phys. Rev. D 59, 075006 (1999).

[4] M. B. Tully and G. C. Joshi, Phys. Lett. B466, 333 (1999); E. M. Gregores, A. Gusso and S. F. Novaes, Phys. Rev. D 64, 015004 (2001); J. L. Garcia-Luna, G. Tavares-Velasco, J. J. Toscano, Phys. Rev. D 69, 093005 (2004).

[5] V. Pleitez and M. D. Tonasse, Phys. Rev. D 48, 2353 (1993).

[6] K. Hagiwara et el., (Particle Data Group), Phys. Rev. D 66, 010001 (2002).

[7] P. H. Frampton, Int. J. Mod. Phys. A13, 2345 (1998), P. H. Frampton, Mod. Phys. Lett. A18, 1377 (2003).

[8] A. G. Dias, R. Martinez, V. Pleitez, hep-ph/0407141. 
[9] J. C. Montero, F. Pisano and V. Pleitez, Phys. Rev. D 47, 2918 (1993); R. Foot, H. N. Long and T. A. Tran, Phys. Rev. D 50, R34 (1994); H. N. Long, Phys. Rev. D 53, 437 (1996);

[10] A. G. Dias, V. Pleitez, and M. D. Tonasse, Phys. Rev. D 67, 095008 (2003); A. G. Dias and V. Pleitez, Phys. Rev. D 69, 077702 (2004); H. N. Long, N. Q. Lan, Europhys. Lett. 68, 571 (2003); A. G. Dias, C. A. de S. Pires, and P. S. Rodrigues da Silva, Phys. Rev. D 68, 115009 (2003); G. Tavares-Velasco and J. J. Toscano, Phys. Rev. D 65, 013005 (2002); C. A. de S. Pires and P. S. Rodrigues da Silva, Eur. Phys. J. C36, 397 (2004); M. A. Perez, G. Tavares-Velasco and J. J. Toscano, Phys. Rev. D 69, 115004 (2004); J. C. Montero, V. Pleitez and M. C. Rodriguez, Phys. Rev. D 70, 075004 (2004); G. Tavares-Velasco and J. J. Toscano, Phys. Rev. D 70, 053006 (2004); J. A. Rodriguez and M. Sher, hep-ph/0407248.

[11] R. Foot, H. Lew, X.-G. He, G. C. Joshi, Z. Phys. C 44, 441 (1989)

[12] J. C. Montero, C. A. de S. Pires and V. Pleitez, Phys. Rev. D 65, 095001 (2002). The electric charge atribution of the lepton octet must be corrected in this work. But it does not affect their subsequent conclusions. 